\title{
$\$$ Research Square

\section{High mortality rate of obstetric critically ill patients in Rwanda and its predictability}

\author{
Alcade Rudakemwa ( $\nabla$ rudakal@gmail.com ) \\ Ruhengeri referral hospital https://orcid.org/0000-0002-5945-6761 \\ Amy Lucille Cassidy \\ Wake Forest University School of Medicine \\ Theogene Twagirumugabe \\ University of Rwanda College of Medicine and Health Sciences Huye
}

Research article

Keywords: Obstetric, intensive care unit, critical care, mortality prediction

Posted Date: December 30th, 2020

DOI: https://doi.org/10.21203/rs.3.rs-29207/v2

License: (c) (1) This work is licensed under a Creative Commons Attribution 4.0 International License.

Read Full License 


\section{Abstract}

Background Reasons for admission at the intensive care units (ICU) for obstetric patients vary from a setting to another. Outcomes from ICU and its prediction models are not well explored in Rwanda because of lack of appropriate scores. This study intended to assess profile and accuracy of predictive models for obstetric patients admitted in ICU in the two public tertiary hospitals in Rwanda.

Methods We prospectively collected data from all obstetric patients admitted in the ICU of public referral hospitals in Rwanda from March 2017 to February 2018 to identify reasons for admissions and factors for prognosis. We analysed the accuracy of mortality prediction models including the quick Sequential Organ Failure Assessment (qSOFA) and Modified Early Obstetric Warning Score (MEOWS) by using the Logistic Regression and adjusted Receiver Operating characteristic (ROC) curves.

Results Obstetric patients represented $12.8 \%$ of all ICU admissions and $1.8 \%$ of all deliveries. Sepsis (31.9\%) and haemorrhage (25.5\%) were the two commonest reasons for ICU admission in our study participants. The overall ICU mortality for our obstetric patients was $54.3 \%$ while the average length of stay was 6.6 days. MEOWS score was an independent predictor to mortality (adjusted OR=1.25[1.071.46]; $p=0.005$ ) and so was the qSOFA score (adjusted $O R=2.81[1.25-6.30] ; p=0.012$ ). The adjusted Area Under the ROC (AUROC) for MEOWS was 0.773[0.666-0.880] and that of the qSOFA was $0.764[0.654-$ 0.873] signing fair accuracies for ICU mortality prediction in these settings for both models.

Conclusion Sepsis is the commonest reason for admissions to ICU for obstetric patients in Rwanda. Simple models comprising MEOWS and qSOFA could accurately predict the mortality for those patients but further larger studies are needed before generalization.

\section{Background}

Various reasons for admission of obstetric patients to the ICU have been identified and the prevalence of each admitting diagnosis varies between countries. Hypertensive disorders and obstetric hemorrhage are predominant among obstetric patients in high and middle income countries, whereas the most common reasons in low income countries in Africa are hemorrhage and sepsis[2,3,5,6].

Mortality among obstetric patients admitted to the ICU remains relatively high in low income countries compared to high income countries. While estimated at 3.5\% in Netherlands, it was almost 10 times higher in Kenya and South Africa[5,7,8]. However, the predicting maternal mortality remains challenging as currently used severity scores are not suitable for obstetric patients admitted to the ICU[9,10]. Recently, other tools have been developed for critically ill obstetric patients like CIPHER (Collaborative Integrated Pregnancy High-dependency Estimated of Risk) and ICNARC (Intensive Care National Audit and Research Centre) with high discrimination but seem challenging in low resource settings with limited laboratory capacities[11,12]. Evidence showed that the Modified Early Obstetric Warning Score (MEOWS) developed by the Confidential Enquiry into Maternal and Child Health (CEMACH) and the quick Sequential Organ Failure Assessment (qSOFA) may help in early detection of physiological derangements[13-15]. 
There is no specific publication on obstetric admissions to ICU and evidence is lacking that these tools may predict outcome for obstetric patients admitted to ICU in Rwanda. Therefore, this study was conducted to determine the reasons for ICU admissions, outcomes of obstetric patients admitted to ICU, and to evaluate the accuracy of MEOWS and qSOFA in the prediction of mortality for obstetric patients admitted to the ICU.

\section{Methods}

The study was conducted in two main teaching hospitals in Rwanda: Centre Hospitalier Universitaire de Butare (CHUB) and Centre Hospitalier Universitaire de Kigali (CHUK) which are tertiary hospitals with total of 448 beds plus 6-bed ICU and 519 bed plus 7-bed ICU respectively. After obtaining ethical approval from the University of Rwanda/College of Medicine and Health Sciences (CMHS) [approval notice No 118 /CMHS IRB/2017], we conducted a prospective cross-sectional study.

We included all women who were admitted to ICU during pregnancy or within 42 days of termination of pregnancy in the study. Patients were followed from admissions to discharge from ICU. A nurse from ICU department collected following data: age, gravida, vital signs, reason for admission, ICU management (duration of stay, inotropes or vasopressors use, blood transfusion, interventions (surgical procedures, ventilation and dialysis) and outcome (mortality or discharge). The duration of stay was calculated in terms of days with zero days for a stay shorter than 24 hours. Vital signs collected were used to manually calculate Modified Early Obstetric Warning System (MEOWS) and quick Sequential Organ Failure Assessment (qSOFA) scores at admission to ICU.

Data were analysed using the Statistical Package for Social Sciences (IBM SPSS Statistics for Windows, version 22.0. Armonk, NY: IBM Corp). Descriptive results were reported as frequency or percentages, mean +/- standard deviation, median and interquartile range (IQR) accordingly. The proportions of obstetric patients admitted to ICU were calculated comparing them to all deliveries reported by both hospitals and all ICU admissions during the study period. Comparison of frequencies or median scores of different variables was made between survivors and non-survivors by using the Chi-square or MannWhitney $U$ tests accordingly. A p-value lower than 0.05 was considered statistically significant. Variables with significant association with the survival rate $(p<0.250)$ were included in a logistic regression model to identify the independent predictors to the mortality. We calculated the discriminatory probability of each prediction model of mortality including either the qSOFA or the MEOWS by using the respective model-based probabilities of mortality to generate adjusted Receiver Operating Characteristic Curves (ROC) and adjusted areas under the ROC (AUROC). Moreover, AUROCs for each individual risk score (unadjusted analysis) are also calculated and curves provided beside the previous. For all AUROC, the $95 \% \mathrm{Cl}$ was calculated.

\section{Results}

Demographic data and severity score of obstetric patients at admission to ICU 
During the study period, 747 patients were admitted to the ICUs of CHUB and CHUK. Of them, 94 (12.8\%) were admitted during or within their 42 days after delivery. These obstetric patients were drawn from 4,999 patients admitted to the labour units in the two facilities, corresponding to $1.8 \%$ of obstetric patients.

Table 1 shows the characteristics of obstetric patients admitted to ICU. The mean age of these patients was $29.8+/-6.5$ years, 52 (55.3\%) were admitted at their first or second pregnancy and $44.7 \%(42)$ at their third or above. 70 (74.5\%) were admitted during the post-partum period, 13 (13.8\%) presented after abortion or ectopic pregnancy whereas $11(11.7 \%)$ were pregnant at the time of their admission to the ICU. Of the 70 patients admitted in post-partum period, 44 (62.9\%) delivered by cesarean section and 26 (37.1\%) had vaginal deliveries.

Table 1. Characteristics of obstetric patients admitted in ICU in our study

\begin{tabular}{llll}
\hline Variables & Range & Frequency & Mean \pm SD \\
\hline Age (in years) & & $29.82 \pm 6.507$ \\
Gravidity at admission to ICU & $1-2$ & $52(55.3)$ \\
& $\geq 3$ & $42(44.7)$ \\
Period of admission & During pregnancy & $11(11.7)$ \\
& Post-abortion/ectopic pregnancy & $13(13.8)$ \\
& Post-partum & $70(74.5)$ \\
Admitted in post-partum period (n=70) & \\
Mode of delivery & Cesarean section & $44(62.9)$ \\
& Normal delivery & $26(37.1)$ \\
\hline
\end{tabular}

Reasons for admission and interventions done

The most common reason for admission to ICU for obstetric patients was sepsis (31.9\%), and then the obstetric hemorrhage (25.5\%), while other diseases including cardiomyopathy, stroke, embolism and trauma represent $20.2 \%$ of admissions. Hypertensive disorders of pregnancy (17.02\%) and malaria (5.3\%) were least represented (table 2).

Table 2: Reasons for admissions and interventions performed on study patients during their ICU stay 


\begin{tabular}{lll}
\hline Variables & Number of patients & Percentage (\%) \\
\hline Reason for ICU admissions & & \\
Hemorrhage & 24 & 25.5 \\
Hypertensive disorders of pregnancy & 16 & 17.0 \\
Sepsis & 30 & 31.9 \\
Malaria & 5 & 5.3 \\
Others & 19 & 20.2 \\
$\quad$ Cardiomyopathy & 5 & 5.3 \\
$\quad$ Embolism & 7 & 7.5 \\
$\quad$ Trauma & 5 & 5.3 \\
$\quad$ Stroke & 2 & 2.1 \\
Interventions done & & \\
Invasive mechanical ventilation & 90 & 95.7 \\
Blood transfusion & 33 & 35.1 \\
Inotropics/vasopressors support & 47 & 50.0 \\
Re-operation & 5 & 5.3 \\
Hemodialysis & 4 & 4.3 \\
\hline
\end{tabular}

Of the various interventions (table 2) received by patients admitted to the ICU, respiratory support by mechanical ventilation was the main intervention (95.7\%), as well as inotropic support or vasopressors' need (50.0\%), blood transfusion (35.1\%), re-operation (5.3\%) and hemodialysis (4.3\%). Some patients received more than one intervention due to severity of disease that required more than one of the above-mentioned interventions.

Statistical analysis of outcome, mortality prediction and length of stay in ICU

The mean length of stay in ICU for our study participants was $6.6 \pm 7.525$ days. During the period of study, 51 of 94 (54.3\%) obstetric patients admitted to ICU died.

Analysis of factors associated with the ICU survival (table 3) showed that setting, mode of delivery and reasons for admission are not associated with survival rate. There were however statistically significant different MEOWS scores between survivors and non-survivors. We found a median MEOWS of 7 (IQR: 6; 8 ) for survivors versus 8 (IQR: 6 ; 12) for non-survivors ( $p=0.001)$. It was also the case for qSOFA with median of qSOFA of $2($ IQR: $1 ; 2)$ in the two groups $(p=0.003)$. Multivariable logistic regression showed that when adjusted for reason for admission and Caesarean section before admission, MEOWS was an independent predictor of mortality with adjusted OR of 1.25[1.07-1.46]; $p=0.005$. Similarly, one point of increase of qSOFA increased odds of ICU mortality by $181 \%$ [adj.OR: 2.81[1.25-6.30];p=0.012) [Table 4].

Table 3. Factors associated with survival rate 


\begin{tabular}{llllll}
\hline Variable & & $\mathbf{N}=\mathbf{9 4}$ & $\begin{array}{l}\text { Survivors } \\
(\%)\end{array}$ & $\begin{array}{l}\mathrm{n} \\
\text { Non-survivors } \\
(\%)\end{array}$ & $\begin{array}{l}\mathrm{n} \text { - } \\
\text { value }\end{array}$ \\
\hline Setting & CHUK & 60 & $25(26.6)$ & $35(37.2)$ & \\
Reason for admission & CHUB & 34 & $18(19.1)$ & $16(17.0)$ & 0.389 \\
& Hemorrhage & 24 & $11(11.7)$ & $13(13.8)$ & 0.078 \\
& Sepsis & 30 & $9(9.6)$ & $21(22.3)$ & \\
Mode of delivery & Others & 40 & $23(24.5)$ & $17(18.1)$ & \\
& C-section & 44 & $24(25.5)$ & $20(21.3)$ & 0.058 \\
MEOWS & Vaginal & 26 & $9(9.6)$ & $17(18.1)$ & \\
(IQR)] & & & $7(6 ; 8)$ & $8(6 ; 12)$ & 0.001 \\
qSOFA & & & & & \\
[Median (IQR)] & & & $2(1 ; 2)$ & $2(1 ; 2)$ & 0.008 \\
\hline
\end{tabular}

Table 4. Multivariable Logistic regression for MEOWS and qSOFA/predictors of ICU mortality

\begin{tabular}{llll}
\hline Variables & & Adjusted OR [95\%CI] & p-value \\
\hline MEOWS & & $1.25[1.07-1.46]$ & 0.005 \\
Caesarean & $0.39[0.12-1.22]$ & 0.106 \\
Reason for admission & Haemorrhage & 1 (Ref) & \\
& Sepsis & $1.72[0.42-6.94]$ & 0.449 \\
& Others & $0.65[0.18-2.39]$ & 0.517 \\
qSOFA & & $2.81[1.25-6.30]$ & 0.012 \\
Caesarean & & $0.33[0.11-1.02]$ & 0.054 \\
Reason for admission & Haemorrhage & 1 (Ref) & \\
& Sepsis & $1.50[0.38-5.93]$ & 0.559 \\
& Others & $0.88[0.24-3.31]$ & 0.855 \\
\hline
\end{tabular}

In the analysis of both mortality prediction models, the adjusted areas under the receiver operation curves (AUROCs) are presented in the figure 1 beside the unadjusted AUROCs for individual MEOWS and qSOFA score. The unadj. AUROC for qSOFA and for WEOWS were 0.662[0.553-0.771] and 0.705[0.6000.811 ] respectively showing rather a fair mortality prediction for MEOWS. The adjusted AUROC for 
MEOWS and qSOFA were 0.773 [0.666-0.880] and $0.764[0.654-0.873]$ signing fair discrimination capacity for mortality prediction with the two models.

\section{Discussion}

This study evaluated the incidence and outcome of obstetric admissions to ICU in public referral hospitals in Rwanda. We also assessed the accuracy of affordable mortality prediction models using tools that are affordable in resource-limited settings. In our findings, obstetric admissions to ICU in public referral hospitals in Rwanda accounted for $12.8 \%$ of all ICU admissions and occur in $1.8 \%$ of all deliveries. These rates of ICU admission for obstetric patients are relatively higher compared with those reported in high income countries $(0.22-0.76 \%[2,16,17]$. They are rather similar to those found in middle income countries like Brazil (1\%) and Turkey (1.27\%)[2,16-19]. Our findings are also comparable to that in a study done in Nigeria where obstetric represented $17.29 \%$ of ICU admissions and $2.05 \%$ of all deliveries[20].

In this study, the rate of ICU admission to all deliveries was $1.8 \%$. It might have been higher given the limited capacity of our ICUs representing only around $1.5 \%$ of hospital beds while the ideal number should be more than $10 \%$ as it is the case in high income countries[21,22]. This challenge of scarcity of ICU beds is shared with other sub-Saharan African countries where that the number of obstetric patients admitted in ICU falls in a range of $0.24-0.97 \%[5,8,23]$. The severity of conditions for our obstetric patients, as evidenced by the rate of ventilation (90\%) and inotropes' use (50\%), precludes that the number of obstetric admissions to ICU in Rwanda could have been increased. Indeed, the two leading causes of admission to ICU for obstetric patients in Rwanda were sepsis (31.9\%) and obstetric haemorrhagic shock (25.5\%). These reasons for admission substantially differ from those prevailing in high income countries to partly explain discrepancies in terms of mortality rates as sepsis and septic shock are generally associated with a high mortality in both high income countries like in United states[24] and low income countries including Rwanda[25]. The second commonest cause of admission is hemorrhagic shock and resulting coagulation disorders related to delays to achieve haemostasis, lack of readily available blood products and massive transfusion when these are available that may also contribute to the high mortality in obstetric patients in low income countries. Similar findings of those main reasons for admission in ICU have been reported in a study conducted in Kenya[5].

The mortality rate in our critically ill obstetric patients was as high as $53.4 \%$ but worse outcome has been seen in other sub-Saharan African countries like Burkina Faso where this mortality reached $60 \%[26]$. This poor outcome of our patients may be attributable to the limited capacity of our ICUs on one hand, and to the severity of illness among those admitted in ICU as explained above on the other hand. The mortality for obstetric patients admitted in ICU from our study is comparable to one for general ICU patients in Rwanda where it was 48.7\%[25]. Data from our study shows that sepsis was highly prevalent and these results correlate with a single centre study conducted in Rwanda where sepsis was the most common causes of morbidity and mortality among obstetric patients admitted in tertiary hospital[27]. 
A number of mortality prediction tools have been developed for general patients admitted in ICU such as the Acute Physiology and Chronic Health Evaluation (APACHE), the Simplified Acute Physiology Score (SAPS) and the Sequential Organ Failure Assessment (SOFA). However, their generalisation to obstetric patients remains challenging[28]. Our study evaluated the accuracy of models including either MEOWS or qSOFA in predicting mortality for obstetric patients admitted to ICU as easy tools and their components are part of routine clinical assessment. Yet, the respective models have good discriminative power with an area under the curve showing their performance (AUROC: $0.773[0.666-0.880]$ for MEOWS model and $0.764[0.654-$ 0.873 ] for the model containing qSOFA score). Similarly, in a study conducted in Australia among emergency patients with suspected sepsis, it was found that a positive qSOFA ( $\geq 2$ points) identified patients at higher risk of in-hospital mortality or longer ICU stay[29]. In a study done in India, the AUROC showed good discriminative power with qSOFA in predicting mortality (AUROC: $0.73 ; 95 \% \mathrm{Cl}, 0.69$ 0.77) among septic patients admitted, both in ICU and non-ICU wards [27]. Above findings have similarities with our study with regards to qSOFA as predictive model, though our findings are applied in obstetric patients. Our findings on the accuracy of MEOWS predictive model are comparable to those in a research conducted in the United Kingdom which showed that MEOWS had high sensitivity and good specificity to early detect morbidity among obstetric patients outside ICU[14]. Though different settings, MEOWS as a simple bedside model may be applied to obstetric patients at admission to ICU to predict their outcome.

Data for this study were prospectively collected from two tertiary hospitals, which may give it strength to be generalizable to whole obstetric population in the country. However, the study has its own limitations such as the small sample size to allow this extrapolation to the general population. To achieve this, it would be necessary to collect data for a longer period given the limited number of ICU and ICU beds in the country[31]. Furthermore, it could have been interesting to follow up those patients after their discharge from ICU to also report the mortality at 28 and 90 days but many of them were discharged before those dates and could not be reached anymore.

\section{Conclusion}

Hemorrhage and sepsis are major reasons of obstetric admissions to ICU in Rwanda. A relatively high maternal mortality was observed among obstetric patients in ICU. The use of MEOWS and qSOFA in simple mortality prediction models may help to early recognize obstetric patients at high risk of poor outcome. Further studies with larger sample size are needed to evaluate these affordable tools in that regard for resource limited settings.

\section{Abbreviations}

adj. OR: Adjusted Odd ratio

APACHE: Acute, Physiology, Age and Chronic Health Evaluation 
AUROC: Area Under the Receiver Operating Characteristic Curve

CEMACH: Confidential Enquiry into Maternal and Child Health

CHUB: Centre Hospitalier Universitaire de Butare

CHUK: Centre Hospitalier Universitaire de Kigali

Cl: Confidence Interval

CMHS: College of Medicine and Health Sciences

Corp.: Corporate

IBM: International Business Machines

ICU: Intensive Care Unit

IQR: Inter-quartile range

MEOWS: Modified Early Obstetric Warning Score

NY: New York

OR: Odd ratio

p-value: calculated Probability

qSOFA: Quick Sequential Organ Failure Assessment

Ref: Reference

ROC: Receiver Operating Characteristic curve

SAPS: Simplified Acute Physiology Score

SCCM: Society of Critical Care Medicine

SD: Standard deviation

SOFA: Sequential Organ Failure Assessment

SPSS: Statistical Package for Social Sciences

\section{Declarations}

Ethics approval and consent to participate 
The study was approved by ethical and scientific committee of the University of Rwanda College of Medicine and Health Sciences (CMHS) [approval notice No 118 /CMHS IRB/2017]. The individual consent for participation was waived given the minimal risk to the patients.

\section{Consent for publication}

Not applicable. The manuscript does not contain individuals' personal data.

\section{Availability of data and materials}

The datasets used during the current study are available from the corresponding author on reasonable request.

\section{Acknowledgements}

We are grateful to Nurses working in the intensive care unit: Mahoro Jean de Dieu of Butare University Teaching Hospital (CHUB) and Twagirimana Uzziel of the Kigali University Teaching Hospital (CHUK).

\section{Competing interests}

No competing interests are declared.

\section{Funding}

No external funding declared.

\section{Authors' contributions}

AR: Study design, data analysis, interpretation and manuscript writing.

TT: Data analysis, interpretation and manuscript writing.

ALC: Study design and manuscript writing.

All authors have read and approved the final manuscript.

\section{References}

1. Pollock W, Dennis C. Pregnant and postpartum admissions to the intensive care unit: a systematic review. Intensive Care Med. 2010;36:1465-74.

2. Keizer JL, Zwart JJ, Meerman RH, Harinck BIJ, Feuth HDM, van Roosmalen J. Obstetric intensive care admissions: A 12-year review in a tertiary care centre. Eur J Obstet Gynecol Reprod Biol. 2006;128(12):152-6. 
3. Leung NYW, Lau a CW, Chan KKC, Yan WW. Clinical characteristics and outcomes of obstetric patients admitted to the Intensive Care Unit: a 10-year retrospective review. Hong Kong Med J. 2010;16(1):18-25.

4. Bendre K, Tuteja T, Niyogi G. Critically ill obstetric patients. Int J Reprod Contraception, Obstet Gynecol [Internet]. 2015;4(2):370. Available from: http://www.scopemed.org/?mno=178109

5. Githae F, Mung'ayi V, Stones W. Course and outcome of obstetric patients admitted to a University Hospital Intensive Care Unit. East Afr Med J [Internet]. 2013;88(10):356-60. Available from: http://www.ajol.info/index.php/eamj/article/view/86834

6. Adeniran AS, Bolaji BO, Fawole AA. Predictors of maternal mortality among critically ill obstetric patients. Malawi Med J. 2015;27(March):16-9.

7. Zwart JJ, Dupuis JRO, Richters A, Ö ry F, Van Roosmalen J. Obstetric intensive care unit admission: A 2-year nationwide population-based cohort study. Intensive Care Med. 2010;36(2):256-63.

8. Ntuli TS, Ogunbanjo G, Nesengani S, Maboya E, Gibango M. Obstetric intensive care admissions at a tertiary hospital in Limpopo Province, South Africa. South African J Crit Care [Internet]. 2015;31(1):8. Available from: http://www.sajcc.org.za/index.php/SAJCC/article/view/164

9. Ryan HM, Sharma S, Magee LA, Ansermino JM, MacDonell K, Payne BA, et al. The Usefulness of the APACHE II Score in Obstetric Critical Care: A Structured Review. J Obstet Gynaecol Canada [Internet]. 2016;38(10):909-18. Available from: http://dx.doi.org/10.1016/j.jogc.2016.06.013

10. Stevens TA, Carroll MA, Promecene PA, Seibel M, Monga M. Utility of Acute Physiology, Age, and Chronic Health Evaluation (APACHE III) score in maternal admissions to the intensive care unit. Am J Obstet Gynecol. 2006;194(5):13-5.

11. Payne BA, Ryan H, Bone J, Magee LA, Aarvold AB, Ansermino JM, et al. Development and internal validation of the multivariable CIPHER ( Collaborative Integrated Pregnancy High-dependency Estimate of Risk ) clinical risk prediction model. Crit Care. 2018;22(278):1-13.

12. Simpson NB, Shankar-hari M, Rowan KM, Cecconi M, Dadelszen P Von, Huning EY, et al. Maternal Risk Modeling in Critical Care- Development of a Multivariable Risk Prediction Model for Death and Prolonged Intensive Care*. Crit Care Med. 2020;48(5):663-72.

13. Paternina-Caicedo A, Miranda J, Bourjeily G, Levinson A, Dueñas C, Bello-Muñoz C, et al. Performance of the Obstetric Early Warning Score in critically ill patients for the prediction of maternal death. Am J Obstet Gynecol [Internet]. 2017;216(1):58.e1-58.e8. Available from: http://dx.doi.org/10.1016/j.ajog.2016.09.103

14. Singh S, McGlennan A, England A, Simons R. A validation study of the CEMACH recommended modified early obstetric warning system (MEOWS). Anaesthesia. 2012;67(1):12-8. 
15. Opal SM, Rubenfeld GD, Poll T Van Der, Vincent J, Angus DC. The Third International Consensus Definitions for Sepsis and Septic Shock (Sepsis-3). 2016;315(8):801-10.

16. Wanderer JP, Leffert LR, Mhyre J, Kuklina E, Callaghan WM, Bateman BT. Epidemiology of Obstetric-related ICU admissions in Maryland: 1999 - 2008. Crit Care Med. 2014;41(8):1844-52.

17. Yi HY, Jeong SY, Kim SH, Kim Y, Choi S, Oh S, et al. Indications and characteristics of obstetric patients admitted to the intensive care unit: a 22-year review in a tertiary care center. Obs Gynecol Sci. 2018;61(2):209-19.

18. Togal T, Yucel N, Gedik E, Gulhas N, Toprak HI, Ersoy MO. Obstetric admissions to the intensive care unit in a tertiary referral hospital. J Crit Care [Internet]. 2010;25(4):628-33. Available from: http://dx.doi.org/10.1016/j.jcrc.2010.02.015

19. Bandeira ARAP, Rezende CAL, Reis ZSN, Barbosa AR, Peret FJA, Cabral AC V. International Journal of Gynecology and Obstetrics Epidemiologic pro fi le, survival , and maternal prognosis factors among women at an obstetric intensive care unit. Int J Gynecol Obstet [Internet]. 2014;124(1):63-6. Available from: http://dx.doi.org/10.1016/j.ijgo.2013.07.015

20. Embu HY, Isamade ES, Nuhu SI, Oyebode TA, Kahansim ML. Obstetric admissions in a general intensive care unit in north-central nigeria. 2016;33(April).

21. Halpern NA, Pastores SM. Critical care medicine in the United States 2000-2005: An analysis of bed numbers, occupancy rates, payer mix, and costs. Crit Care Med. 2010;38(1):65-71.

22. Murthy S, Leligdowicz A, Adhikari NKJ. Intensive care unit capacity in low-income countries: A systematic review. PLoS One [Internet]. 2015;10(1):1-12. Available from:

http://dx.doi.org/10.1371/journal.pone.0116949

23. Green K, Orazulike N. Obstetric Admission into the Intensive Care Unit (ICU) of the University of Port Harcourt Teaching Hospital: A Ten-Year Review. J Adv Med Med Res. 2018;25(9):1-7.

24. Hajj J, Blaine N, Salavaci J, Jacoby D. The "Centrality of Sepsis": A Review on Incidence, Mortality, and Cost of Care. Healthcare [Internet]. 2018;6(3):90. Available from: http://www.mdpi.com/2227$9032 / 6 / 3 / 90$

25. Riviello ED, Kiviri W, Fowler RA, Mueller A, Novack V, Banner-Goodspeed VM, et al. Predicting mortality in low-income country icus: The Rwanda mortality probability model (R-MPM). PLoS One. 2016;11(5):1-14.

26. Dao B, Rouamba A, Ouédraogo D, Kambou T, Bazié AJ. Transfert de patientes en état gravidopuerpéral en réanimation: À propos de 82 cas au Burkina Faso. Gynecol Obstet Fertil. 2003;31(2):123-6. 
27. Rulisa S, Umuziranenge I, Small M, Roosmalen J Van. Maternal near miss and mortality in a tertiary care hospital in Rwanda. BMC Pregnancy Childbirth [Internet]. 2015;1-7. Available from: http://dx.doi.org/10.1186/s12884-015-0619-8

28. Id KA, Souza RD, Pinto R, Ray JG, Hill A, Scales DC, et al. Risk prediction models for maternal mortality: A systematic review and meta-analysis. 2018;823:1-20.

29. Canet E, Taylor DM, Khor R, Bellomo R. qSOFA as predictor of mortality and prolonged ICU admission in Emergency Department patients with suspected infection. J Crit Care [Internet]. 2018;\#pagerange\#. Available from: https://doi.org/10.1016/j.jcrc.2018.08.022

30. Maitra S, Som A, Consultant A, Bhattacharjee S, Resident S. Accuracy of quick Sequential Organ Failure Assessment (qSOFA) score and systemic inflammatory response syndrome (SIRS) criteria for predicting mortality in hospitalized patients with suspected infection: A meta-analysis of observational studies. Clin Microbiol Infect [Internet]. 2018; Available from: https://doi.org/10.1016/j.cmi.2018.03.032

31. Munyiginya P, Brysiewicz P, Mill J. Critical care nursing practice and education in Rwanda. South African J Crit Care. 2016;32(2):55-7.

\section{Figures}
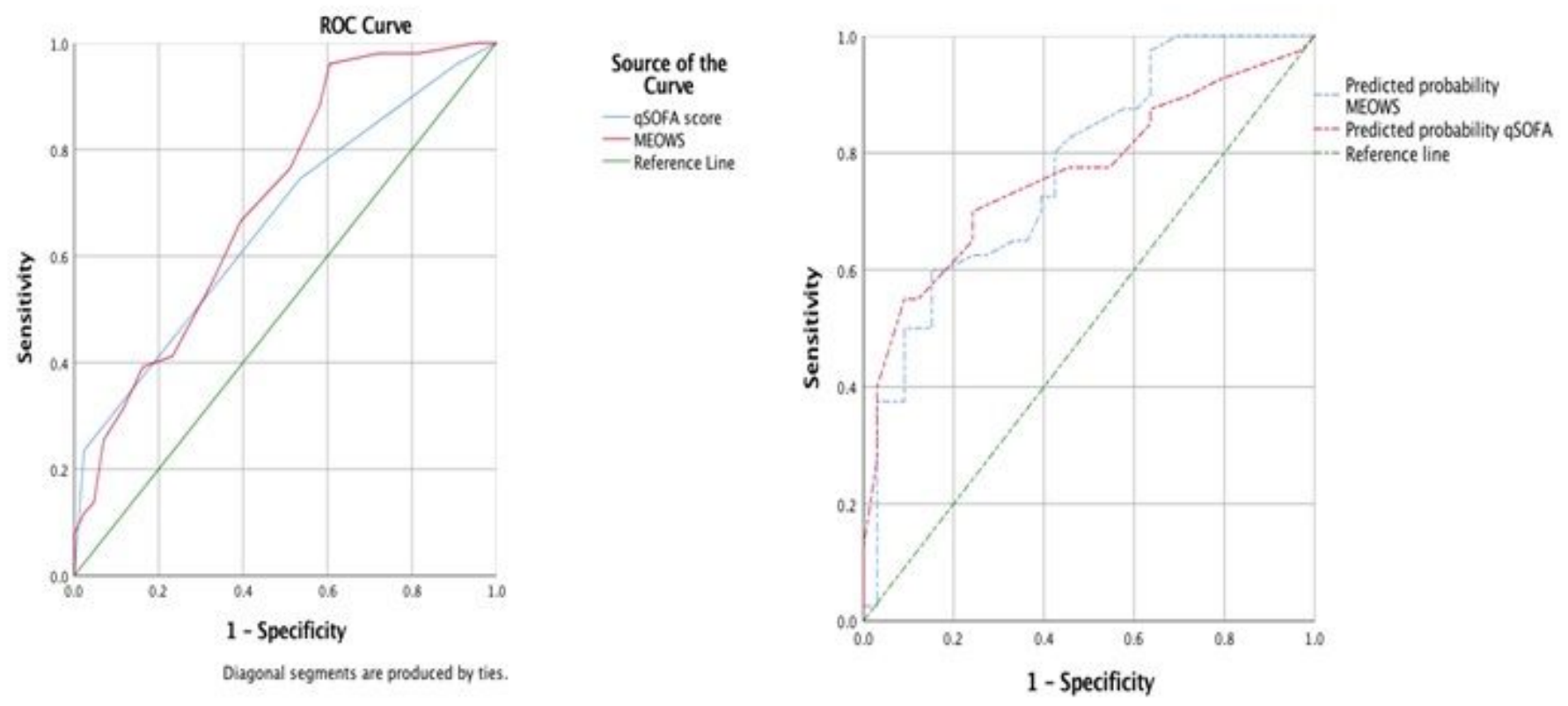

\section{Figure 1}

Unadjusted and adjusted AUROC for prediction of ICU mortality by qSOFA and MEOWS 